\title{
Exact, Scaled Image Rotation Using the Finite Radon Transform
}

\author{
Imants Svalbe \\ School of Physics, \\ Monash University, \\ Australia
}

\begin{abstract}
In traditional tomography, a close approximation of an object can be reconstructed from its sinogram. The orientation (or zero angle) of the reconstructed image can be chosen to be any one of the many projected view angles. The Finite Radon Transform (FRT) is a discrete analogue of classical tomography. It permits exact reconstruction of an object from its discrete projections. Reordering the discrete FRT projections is equivalent to an exact digital image rotation. Each FRT-based rotation preserves the intensity of all original image pixels and allocates new pixel values through use of an area-preserving, anglespecific interpolation filter. This approach may find application in image rotation for feature matching, and to improve the display of zoomed and rotated images.
\end{abstract}

\section{Introduction}

A comparison of the artefacts produced by classic image rotation algorithms appears in 1]. The lack of a unique prescription to interpolate data on a $2 \mathrm{D}$ or higher dimension grid means image rotations, at some angles more than others, can lead to significant errors in the image intensities of the rotated image, especially after multiple, sequential rotations. The mapping of patterns onto grids is a problem well understood by those working in crafts such as tiling and tapestry.

We present here a method for the exact rotation of digital image data based on the Finite Radon Transform (FRT) [2]. The FRT generates a sinogram-like set of discrete projections, $R(t, m)$, from a discrete image, $I(x, y)$. The original data $I(x, y)$ can always be reconstructed exactly from the FRT projections $R(t, m)$. Translating an image, by offset $\left(x_{d}, y_{d}\right)$, in FRT space is almost as simple as translation in image space. $R(t, m)$ gets re-mapped to $R\left(t^{\prime}, m\right)$, where $t^{\prime}=x_{d}+$ $m y_{d}$. However to perform an image rotation in FRT space, $R(t, m)$ must be mapped to a new projection set, $R\left(t^{\prime}, m^{\prime}\right)$. This paper shows how to use the $t$ and $m$ values from a known set of FRT projections of an image to find the appropriate new $t^{\prime}$ and $m^{\prime}$ values that produce an exact, rotated version of the original image data. As the FRT space is an exact mapping of an image space, the same exact rotations can be performed in either image or FRT coordinates. Rotated pixels are treated as lattice points except for up-scaled rotation where, for interpolation, the pixels are treated as squares of unit area. 
The FRT has been used as an alternative approach to the traditional backprojection or Fourier inversion methods to reconstruct real tomographic x-ray projection data [3]. An overview of the properties of FRT projections, relevant to the present work, appears in 4]. Early work on skewing and primitive rotation of images in FRT space, for images on square and hexagonal lattices, appeared in [5. The use of the "natural angles" that occur on a discrete grid is central to this topic. The distribution and frequency of occurrence of the natural angles on discrete grids for the discrete Hough transform, a close relative of discrete Radon projections, was derived in [6]. An extended review of the FRT and its relation to other discrete projection methods is given in [7. Asymmetric sets of projection data occur frequently in tomography, often through practical restrictions on the range of available acquisition angles. The FRT provides a means to symmetrise these projections and hence reconstruct images from asymmetric data sets [8]. The discrete Mojette projective transform [10] is closely related to the FRT. A recent, comprehensive review of the Mojette transform, its relationship to

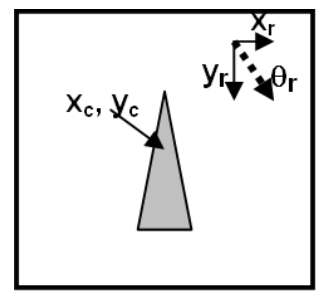

(a)

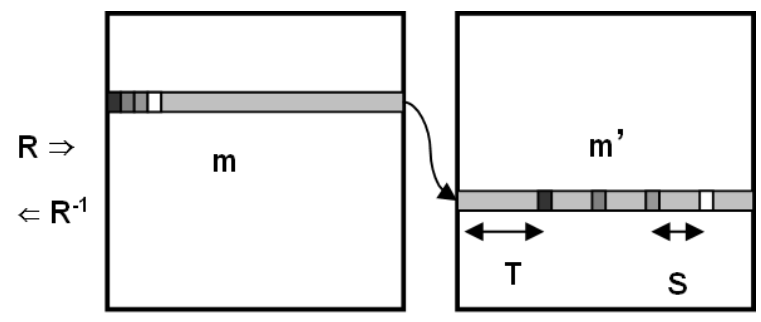

(b)

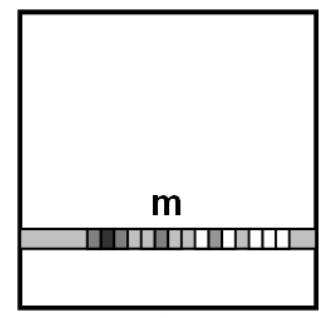

(d) (c)

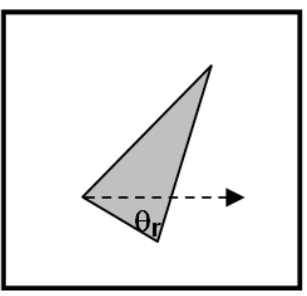

(e)

Fig. 1. Algorithm summary: a) Original image embedded into a larger (pxp) space, $I(x, y)$, with rotation centre $\left(x_{c}, y_{c}\right)$, and rotation vector, $\left(x_{r}, y_{r}\right)$. b) Applying the FRT forward projection operator, $R$, to $I(x, y)$ produces $R(t, m)$, comprised of $p+1$ projection angles, and with each projection containing $\mathrm{p}$ translates. Only one row (labelled $m$ ) of $R(t, m)$ is shown. c) The rotation is performed by re-ordering all rows of the FRT (mapping each $m$ to a new $m^{\prime}$ ) and then shifting (by operator $T$ ) and shuffling (by operator $S$ ) the content of each row to effect the rotation and scale $(S$ and $T$ map $t$ to $t^{\prime}$ ). d) The re-mapped rows are convolved with a linear filter, $f(t)$, to interpolate values for the empty pixels on the new, rotated lattice. e) Applying the inverse FRT operator, $R^{-1}$, reconstructs the original data as a rotated and up-scaled image, $I^{\prime}(x, y)$. 
the FRT and a comprehensive survey of applications for discrete projected data is given in [11]. Kingston, in chapter 6 of [11, showed how to generate multiscale versions of a discrete image from its Mojette-projected data. That work prompted this application to scaled rotations using the FRT formalism.

Figure 1 shows an overview of the FRT-based rotation algorithm presented here. Section 2 gives a short review of the FRT. Section 3 details the step-by-step operation of the FRT rotation algorithm, including a comparison to a real space implementation of the same process. Section 4 provides some image rotation examples. Section 5 outlines possible extensions to this approach and points to areas where it might find application. A summary and conclusions are given in Section 6 .

\section{Finite Radon Transform (FRT)}

The FRT, $R(t, m)$, maps discrete $2 \mathrm{D}$ image intensities, $I(x, y)$, into a set of $1 \mathrm{D}$ discrete projections. $R(t, m)$ is defined by

$$
R(t, m)=\sum_{m=0}^{p-1} \sum_{t=0}^{p-1} \sum_{y=0}^{p-1} I\left(\langle t+m y\rangle_{p}, y\right),
$$

where $\langle t+m y\rangle_{p}$ means the value for $x$ is computed modulus $p$. The FRT formalism extends naturally to discrete projections in $\mathrm{nD}$. The FRT always projects data from an image space that has a prime number, $p$, of rows and the same prime number, $p$, of columns. Each FRT projection is a 1D projected view of the object. The translate position along the $1 \mathrm{D}$ projection is labelled by an integer, $t$. Each FRT projection has the same width, $p, 0 \leq t<p$. Each projection is oriented at an angle labelled by an integer, $m$, there are always $p+1$ FRT projection angles, $0 \leq m \leq p$. Rectangular or irregularly shaped images need to be embedded (usually by zero-padding) into a sufficiently large $p \times p$ image space, in order to apply the FRT.

Displacements in the image space are taken here to increase from left to right for $x$ and from top to bottom for $y$, with $0 \leq x, y \leq p-1$. The position $(x, y)$ of any image pixel that gets summed as part of the projected ray with translate $t$ in the projection at angle $m$ is given by

$$
x=t+m y \text {. }
$$

For $m=p$, the summations are taken along the rows, $R(t, p)=\sum_{x=0}^{p-1} I(x, t)$. For $m=0$, the sums are taken along columns of the image, $R(t, 0)=\sum_{y=0}^{p-1} I(t, y)$.

Following (2), for pixels on a uniform, square lattice, we write, where $\alpha$ is an integer,

$$
m=\left(\alpha p+x_{m}\right) / y_{m}
$$

The values of $x_{m}$ and $y_{m}$ are chosen to minimise $x_{m}^{2}+y_{m}^{2}$. This choice is equivalent [4] to projecting across the image space at the angle $\theta_{m}$ that connects pixels that are nearest neighbours for any given value of $t$. 
The FRT projections span the angle range from 0 to $\pi$ through the four-fold symmetry based on $x_{m}: y_{m}, y_{m}: x_{m},-y_{m}: x_{m}$ and $-x_{m}: y_{m}$, corresponding to $\theta_{m}, \pi / 2-\theta_{m}, \pi / 2+\theta_{m}$ and $\pi-\theta_{m}$ respectively. The projection angle for $m^{\prime}=p-m, \theta_{p-m}$, corresponds to $180-\theta_{m}$. Similarly, $\pi / 2+\theta_{m}$ and $\pi / 2-\theta_{m}$ have complemented $\mathrm{m}$ values. A list of $\mathrm{m}$ values and their associated discrete projection angles for $p=17$ appears in Table 1 . The algorithm described in the next section always chooses one of the $p+1$ "natural" FRT angles from a $p \times p$ grid as the input rotation angle, $x_{r}: y_{r}$. The integer values of $x_{m}$ and $y_{m}$, for a square lattice, range from 0 up to $\sqrt{2 p / \sqrt{3}}$ [7]. There is almost complete overlap in the discrete angle sets that arise from different primes. This very useful property of the FRT is discussed in greater detail in [4].

Table 1. Discrete FRT projection angles for $p \times p$ images with $p=17$. Only half of the $p+1$ values of $m$ are shown.

\begin{tabular}{c|cccccccccc}
$m$ & 0 & 1 & 2 & 3 & 4 & 5 & 6 & 7 & 8 & 9 \\
\hline$x_{m}: y_{m}$ & $0: 1$ & $1: 1$ & $2: 1$ & $3: 1$ & $4: 1$ & $-2: 3$ & $1: 3$ & $-3: 2$ & $-1: 2$ & $1: 2$
\end{tabular}

Table 2. $x_{f}: y_{f}$ is the result of a discrete rotation, $x_{r}: y_{r}=3: 2$ applied to the FRT angles $x_{m}: y_{m}$ for $p=17$, as given in Table 1

\begin{tabular}{c|cccccccccc}
$m$ & 0 & 1 & 2 & 3 & 4 & 5 & 6 & 7 & 8 & 9 \\
\hline$x_{f}: y_{f}$ & $-2: 3$ & $1: 5$ & $4: 7$ & $7: 9$ & $10: 11$ & $-12: 5$ & $3: 11$ & $0: 1$ & $1: 0$ & $-1: 8$
\end{tabular}

\section{Rotation Algorithm}

If a discrete angle, $x_{m}: y_{m}$, is rotated by a discrete angle, $x_{r}: y_{r}$, then the result is a new discrete angle, $x_{f}: y_{f}$, given by

$$
x_{f}: y_{f}=\left(x_{m} x_{r}-y_{m} y_{r}\right):\left(x_{m} y_{r}+x_{r} y_{m}\right) \text {. }
$$

This follows from the definition of $\theta_{i}$ as $\tan ^{-1}\left(y_{i} / x_{i}\right)$ and from $\tan \left(\theta_{m}+\theta_{r}\right)=$ $\left(\tan \theta_{m}+\tan \theta_{r}\right) /\left(1-\tan \theta_{m} \tan \theta_{r}\right)$. Here we take 1:0 to be $0^{\circ}$, pointing along the $+x$ direction, and $0: 1$ to be $90^{\circ}$, pointing along the $+y$ direction. We take $a x_{i}: a y_{i}$ to be the same angle as $x_{i}: y_{i}$. For real projections, $\pi-\theta_{r}$ is equivalent to $+\theta_{r}$, provided that effects such as the hardening of a polychromatic beam can be neglected. For rotations, these angles are distinct. Table 2 shows the angles, $x_{f}: y_{f}$, that result, using (4), when applying a rotation of $x_{r}: y_{r}=3: 2$ to the original set of $x_{m}: y_{m}$ FRT angles, as given in Table 1, for $p=17$.

\subsection{Mapping FRT Rotation $m$ to New Value $\boldsymbol{m}^{\prime}$}

After a rotation by $x_{r}: y_{r}$, some of the resulting rotated FRT discrete angles, as given by (4), do not appear in the original list of nearest neighbour projections 
for that $p$. However, each $m$ value has a multiplicity of potential projection angles. All members of the rotated angle set can be mapped back into one of the known existing angles for that $p$. This cyclic mapping of FRT angle $m$ into the corresponding rotated angle $m^{\prime}$ can be done directly, by re-writing (3) as

$$
m^{\prime}=\left(\alpha^{\prime} p+x_{f}\right) / y_{f},
$$

where $\alpha^{\prime}$ is some integer. Table 3 gives the mapping of $m$ to $m^{\prime}$ for a $3: 2$ rotation of the FRT projection set for $p=17$.

Table 3. Mapping of $m$ to $m^{\prime}$ under a $3: 2$ rotation for $p=17$

\begin{tabular}{l|llllllllllllllllll}
$m$ & 0 & 1 & 2 & 3 & 4 & 5 & 6 & 7 & 8 & 9 & 10 & 11 & 12 & 13 & 14 & 15 & 16 & 17 \\
\hline$m^{\prime}$ & 5 & 7 & 3 & 14 & 4 & 1 & 9 & 17 & 11 & 2 & 16 & 6 & 0 & 13 & 15 & 8 & 12 & 10
\end{tabular}

\subsection{Re-centring FRT Projection $m^{\prime}$}

The position of the pixel $\left(x_{c}, y_{c}\right)$ about which the image rotates determines the translate index $t_{c}$ for the ray from projection $m$ that passes through the image centre. Let the centre point of the original image space be the point $\left(x_{0}, y_{0}\right)$, with translate label $t_{0}$. Usually this point is the geometric centre of the image space, $x_{0}=y_{0}=p / 2$. Following (2),

$$
t_{0}=x_{0}-m y_{0} \quad \text { and } \quad t_{c}=x_{c}-m^{\prime} y_{c}
$$

Then the shift, $T$, required to adjust a projection for a change from FRT row $m$ to FRT row $m^{\prime}$, as a consequence of the rotation, is given by

$$
T=x_{c}-x_{0}+m y_{0}-m^{\prime} y_{c}
$$

If $\left(x_{0}, y_{0}\right)=\left(x_{c}, y_{c}\right)$, then

$$
T=y_{0}\left(m-m^{\prime}\right) \text {. }
$$

Note that the centring operation depends only on the difference between $m$ and $m^{\prime}$ and the location of the rotation centre.

\subsection{Shuffling the $m^{\prime}$ Data to Preserve Translation Order}

The pixel (we will label it as $N$ ) that was located immediately to the right of the centre pixel in the original image need to be located immediately to the right of the centre pixel in the new, rotated frame of reference. A unit increase in translate index for FRT row $m$ requires that the same pixel $N$ be found shifted by $x_{r}$ pixels in the $x$ direction and $y_{r}$ pixels in the $y$ direction. Then the required index, $S$, to correctly shuffle the data is given by 


$$
\begin{gathered}
t_{N^{\prime}}=x_{c}+x_{r}-m^{\prime}\left(y_{c}+y_{r}\right)=t_{N}+x_{r}-m^{\prime} y_{r} \\
\text { so that } \quad t_{N^{\prime}}-t_{N}=x_{r}-m^{\prime} y_{r} .
\end{gathered}
$$

Because the pixel $N$ has to be the translate next to the origin (a translate increment of one), then $S\left(x_{r}-m^{\prime} y_{r}\right)=1$, giving

$$
S=\frac{1}{\left(x_{r}-m^{\prime} y_{r}\right)}
$$

Note that the shuffling of the row data depends only on the new row index $m^{\prime}$ and the rotation angle, $x_{r}: y_{r}$.

\subsection{Convolution of the FRT Row Data}

The expansion of the pixel size upon rotation is compensated for by convolving each row of $R\left(t, m^{\prime}\right)$ with a linear filter, $f(t)$. This filter fills the gaps between the re-sized pixels. The original pixels are zoomed from a square of unit area, to a new pixel of length $\sqrt{\left(x_{r}^{2}+y_{r}^{2}\right)}$ on each side, with a new pixel area, $A$, where $A=x_{r}^{2}+y_{r}^{2}$. The pattern of interpolation weights is shown in Figure 2 for a rotation of $3: 2$. The area of the enlarged pixel is subdivided uniquely into proportional contributions that come from the original, unit area pixels. The only assumption made here is that the original image intensity can be distributed on the basis of the area that each original pixel intersects with the rotated lattice pixels (a uniform intensity pixel model). Integer weights are used to avoid rounding or truncation of intensities during the scaling process. Discrete 2D convolutions in the image space $I(x, y)$ can be implemented as $1 \mathrm{D}$ convolutions in the equivalent $R(t, m)$ FRT space [2].

Table 4 shows some example 1D FRT filter coefficients derived from the 2D weights given in Figure 2, for a $3: 2$ rotation at the new, rotated row index $m^{\prime}$ for $m^{\prime}=0,1$ and 2 . These filters are easy to implement as $1 \mathrm{D}$ cyclic convolutions of length $p$. The 1D FRT filter coefficients vary for each angle $m$. Because the coefficients have reflective symmetry, FRT angles $p-m$ and $m$ share the same $1 \mathrm{D}$ coefficient set. Alternatively, sequential, shifted convolutions can be applied directly, using the coefficients on each individual row of the $2 \mathrm{D}$ filter, one row at a time. When they become long enough to wrap, the summed $1 \mathrm{D}$ filter coefficients add linearly, modulus $p$.

\subsection{Implementing Rotations in FRT Space vs. Image Space}

The aim of this paper was to show the "discrete sinogram" of the FRT can be reformatted to re-define the orientation of discrete reconstructed image data. The same discrete rotations can, of course, be implemented directly in image or Fourier space, without recourse to the FRT, because the image space and its FFT and FRT are one to one mappings. A real space algorithm similar to the present work, but with de-scaling of the rotated image, was presented in [12]. 


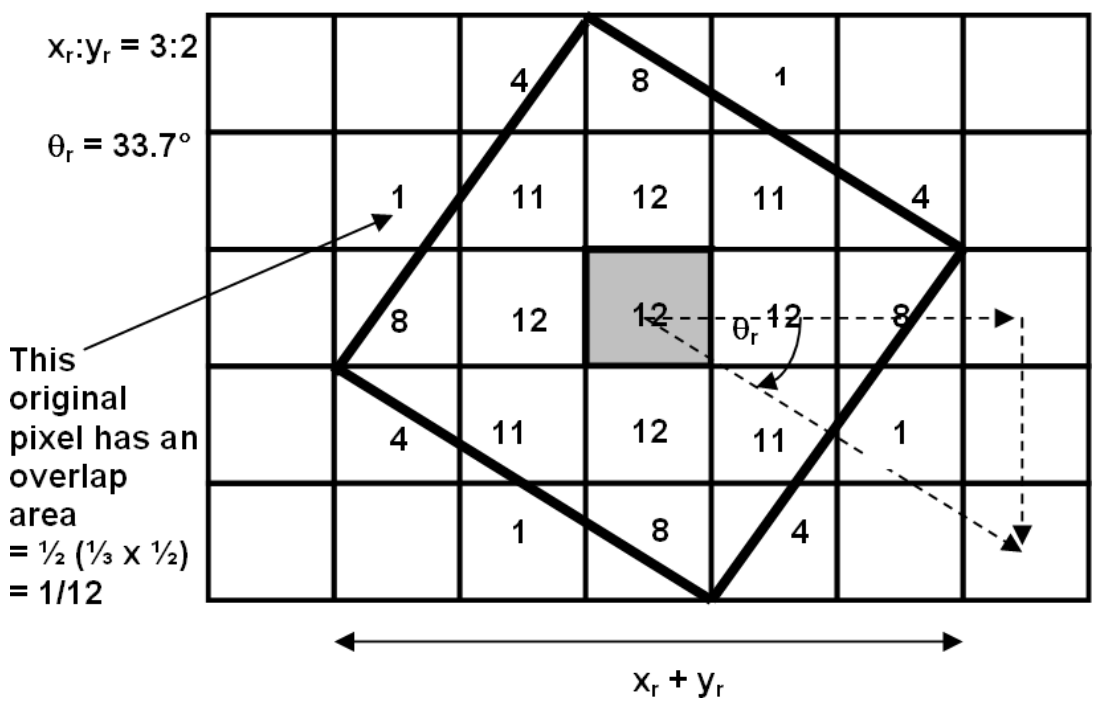

Fig. 2. A unit square (shown shaded) is rotated by the discrete angle $3: 2$ to create an enlarged pixel (with a border shown by the bold lines) which has an area 13 times larger $\left(2^{2}+3^{2}=13\right)$ than the original pixel. The intersection of the zoomed and rotated pixel with the original grid uniquely defines the pixel interpolation weights (given by the integer entry in each original unit square). The FRT projection of these interpolation weights onto the row containing the centre pixel gives the equivalent $1 \mathrm{D}$ linear interpolation filter coefficients to apply on each row $m$ of the FRT space. The sum of the 2D weights shown here is 156 .

Table 4. Interpolation filter weights for a rotation of $3: 2$. The $1 \mathrm{D}$ circular convolution of FRT row data has a different set of weights for each value of $m$. The $1 \mathrm{D}$ filters for FRT angle $p-m$ is the same as that for $m$. The weights for row $m$ are discrete FRT projections, at FRT angle $m$, of the 2D interpolation weights given in Figure 2. The centre pixel of the 1D FRT interpolation filter is shown in bold font. The 1D weights shown here each sum to 156 .

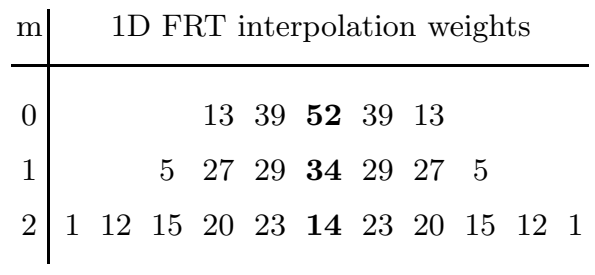

Indeed, the specific 1D convolution applied to each FRT row by the pixel interpolation weights (as shown in Table 5) may be easier to perform directly as a single $2 \mathrm{D}$ convolution in the image space, after applying the inverse FRT operation to reconstruct the rotated original image pixels. 
On the other hand, the $2 \mathrm{D}$ rotation of the image pixels in image space is more easily performed as a simple re-assignment of $\mathrm{m}$ values and shuffling of each row in FRT space. The cost of performing the FRT (and its inverse) scales as $O\left(p^{3}\right)$, or as $O\left(p^{2} \log _{2} p\right)$ if a Fourier based method is used [7]. The overhead of performing the forward FRT on the original, zero-padded image data can be reduced substantially by summing only over the non-zero parts of the oversized pxp image space. Similarly, the FRT space can be sampled to reconstruct only non-zero pixels in the output image, although the scaled, rotated image is usually chosen to nearly fill the pxp space. The FRT rotation method has a clear advantage if the application, such as feature matching, is done using projection rather than real space data.

\section{Examples}

The input image used here is a $64 \times 64$ subset of the Lena image. Figure 3 shows the result after example rotations of $2: 3$ and $8: 1$ respectively.

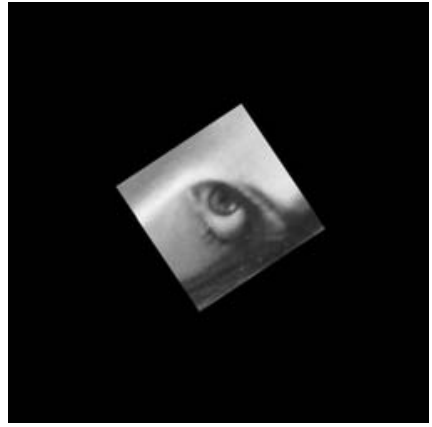

(a)

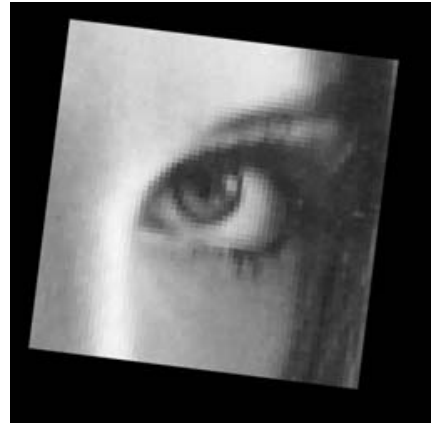

(b)

Fig. 3. Rotation by a) $2: 3\left(\theta_{r}=56.3^{\circ}\right)$ and b) by $8: 1\left(\theta_{r}=7.13^{\circ}\right)$. The periodicity of the FRT means the image will wrap if the rotated image exceeds the size of the initial $p \times p$ array. The final image size here is $647 \times 647$.

\section{$5 \quad$ Applications and Extensions}

The combination of tying image rotation to zoom or scale changes has advantages and disadvantages. The integer scaling factors $x_{r}: y_{r}$ do not need to be large to achieve fine angle rotations. Choosing $x_{r}$ and $y_{r} \leq 10$ defines 32 distinct angles, with a mean spacing of $1.4^{\circ}$ over the range 0 to $\pi / 4$. The image size $(p)$ must be able to be up-scaled by the factor $x_{r}+y_{r}$ for a rotation of $x_{r}: y_{r}$. This scale factor ranges from 2 for $1: 1$, through to 19 for $9: 10$. The image pixel area increases by $x_{r}^{2}+y_{r}^{2}$, ranging from 2 for $1: 1$, through to 181 for $9: 10$. Using signed integers less than 11 for $x_{r}$ and $y_{r}$ provides a choice of 256 distinct rotation angles over 0 to $2 \pi$. 
Table 5. Interpolation of a single pixel after successive rotations. Here a rotation of $3: 2$ is followed by a rotation of $2:-1$. These sequential rotations should be equivalent to a direct rotation of $8: 1$. The original centre pixel is shown in bold. Note the asymmetry in interpolation values. The dotted box encloses the coefficients that should correspond to the direct rotation.

\begin{tabular}{cc|ccccccccc|cc}
0 & 0 & 0 & 0 & 0 & 1 & 3 & 1 & 0 & 0 & 0 & 0 & 0 \\
0 & 0 & 8 & 2 & 4 & 11 & 4 & 3 & 4 & 12 & 4 & 0 & 0 \\
0 & 4 & 12 & 28 & 3 & 25 & 14 & 34 & 23 & 16 & 12 & 0 & 0 \\
0 & 12 & 16 & 20 & 36 & 44 & 45 & 44 & 37 & 20 & 28 & 8 & 0 \\
0 & 0 & 23 & 37 & 47 & 48 & 47 & 45 & 47 & 36 & 32 & 24 & 0 \\
1 & 3 & 34 & 44 & 45 & 48 & 48 & 48 & 48 & 44 & 25 & 11 & 1 \\
3 & 4 & 14 & 45 & 47 & 48 & 48 & 48 & 47 & 45 & 14 & 4 & 3 \\
1 & 11 & 25 & 44 & 48 & 48 & 48 & 48 & 45 & 44 & 34 & 3 & 1 \\
0 & 24 & 32 & 36 & 47 & 45 & 47 & 48 & 47 & 37 & 23 & 4 & 0 \\
0 & 8 & 28 & 20 & 37 & 14 & 45 & 44 & 36 & 20 & 16 & 12 & 0 \\
0 & 0 & 12 & 16 & 23 & 34 & 14 & 25 & 32 & 28 & 12 & 4 & 0 \\
0 & 0 & 4 & 12 & 4 & 3 & 4 & 11 & 4 & 2 & 8 & 0 & 0 \\
0 & 0 & 0 & 0 & 0 & 1 & 3 & 1 & 0 & 0 & 0 & 0 & 0
\end{tabular}

Table 6. Interpolation weights for a single pixel after a direct rotation of $8: 1$

\begin{tabular}{|ccccccccc|}
\hline 1 & 15 & 13 & 11 & 9 & 7 & 5 & 3 & 1 \\
3 & 16 & 16 & 16 & 16 & 16 & 16 & 16 & 15 \\
5 & 16 & 16 & 16 & 16 & 16 & 16 & 16 & 13 \\
7 & 16 & 16 & 16 & 16 & 16 & 16 & 16 & 11 \\
9 & 16 & 16 & 16 & $\mathbf{1 6}$ & 16 & 16 & 16 & 9 \\
11 & 16 & 16 & 16 & 16 & 16 & 16 & 16 & 7 \\
13 & 16 & 16 & 16 & 16 & 16 & 16 & 16 & 5 \\
15 & 16 & 16 & 16 & 16 & 16 & 16 & 16 & 3 \\
1 & 3 & 5 & 7 & 9 & 11 & 13 & 15 & 1 \\
\hline
\end{tabular}

For particular rotations, the same image scale factors arise from different rotation angles. For example, $1: 8$ and $4: 7$ have a common image area zoom of $65(65=12+82=42+72)$. This property occurs [4] only for integers that can be written as the product of primes $p$ where $p=4 n+1$ for some integer $n$, for example, 5,13 and 17 . Here $65=5 \times 13$.

A second rotation treats the newly interpolated values and the original pixels both as "original data" and remaps them (correctly) for the new angle. Performing a rotation of $\theta_{r}$ then $-\theta_{r}$ preserves the original data, but the final interpolated image values depend on the intermediate value of $\theta_{r}$. The interpolation 
Table 7. Interpolation weights for a single pixel after a rotation of $2: 1$, followed by a de-rotation of $2: 1$. These sequential rotations correspond to up-scaling the image pixel area by a factor of 5 in $x$ and $y$. The original centre pixel is shown in bold. Note the asymmetry in interpolation values. The $5 \times 5$ dotted box encloses the coefficients that mimic an up-scaling of an image by a factor of 5 .

\begin{tabular}{ll|ccccccc}
0 & 0 & 0 & 0 & 1 & 3 & 1 & 0 & 0 \\
0 & 0 & 3 & 9 & 6 & 4 & 3 & 0 & 0 \\
1 & 3 & 6 & 10 & 12 & 6 & 10 & 3 & 0 \\
3 & 4 & 6 & 13 & 15 & 13 & 12 & 9 & 0 \\
1 & 6 & 10 & 15 & $\mathbf{1 6}$ & 15 & 10 & 6 & 1 \\
0 & 9 & 12 & 13 & 15 & 13 & 6 & 4 & 3 \\
0 & 3 & 10 & 6 & 12 & 10 & 6 & 3 & 1 \\
0 & 0 & 3 & 4 & 6 & 9 & 3 & 0 & 0 \\
0 & 0 & 1 & 3 & 1 & 0 & 0 & 0 & 0
\end{tabular}

filter for $\theta_{r}$ is the symmetric reflect of the filter for $-\theta_{r}$. The small differences in the images are best shown by the effects on interpolation for a single pixel.

Table 5] shows the interpolated pixel weights from up-scaling each pixel in the input image after a $3: 2$ rotation, followed by a $2:-1$ rotation, corresponding to a one-step rotation of $8: 1$. Table 6 shows the "correct" interpolation weights for a single step $8: 1$ rotation. Table 7 shows the effect of interpolation on a single pixel for a rotation of $2: 1$ followed by de-rotation of 2:-1.

\section{Conclusions}

This work demonstrated exact digital image rotation through a scheme that re-orders the discrete sinogram of projections defined under the FRT formalism. The synchronised scaling and rotation of image pixels preserves the exact, original pixel values, even after multiple successive rotations. Sub-sampling an image back to the original size recovers the original data exactly. The filter coefficients to interpolate the pixel intensities that lie between the zoomed and rotated original values are fixed by the selected discrete rotation angle.

Practical real and FRT space algorithms are given to implement the digital rotation scheme. The FRT implementation of discrete rotations will be an optimal approach for projection-based image processing operations. Manipulation of discrete rotations on grids is an important tool in the reconstruction of images from real projection data.

The algorithm, described here for square image grids, can be adapted for discrete projections on hexagonal lattices. On hexagonal lattices [45], the $x_{m}$ : $y_{m}$ FRT projection $m$ values are symmetric about $0, \pi / 3,2 \pi / 3$ and $\pi$. Another application of simultaneous scale and rotations may be to model the physical flow of vectors from discrete vortices on real lattices. 


\section{References}

1. Danielsson, P.E., Hammerin, M.: High-accuracy rotation of images. Graphical Models and Image Processing (CVGIP) 54(4), 340-344 (1991)

2. Matúš, F., Flusser, J.: Image representation via a finite Radon transform. IEEE Transactions on Pattern Analysis and Machine Intelligence 15(10), 996-1006 (1993)

3. Svalbe, I., van der Spek, D.: Reconstruction of tomographic images using analog projections and the digital Radon transform. Linear Algebra and Its Applications 339, 125-145 (2001)

4. Svalbe, I.: Sampling properties of the discrete Radon transform. Discrete Applied Mathematics 139(1-3), 265-281 (2004)

5. Svalbe, I.: Image operations in discrete Radon space. In: Proc. of the Sixth Digital Image Computing Techniques and Applications, Dicta 2002, pp. 285-290 (2002)

6. Svalbe, I.: Natural representations for straight lines and the hough transform on discrete arrays. IEEE Transactions on Pattern Analysis and Machine Intelligence 11(9), 941-950 (1989)

7. Kingston, A., Svalbe, I.: Projective transforms on periodic discrete image arrays. Advances in Imaging and Electron Physics 139, 75-177 (2006)

8. Chandra, S., Svalbe, I.: A method for removing cyclic artefacts in discrete tomography using Latin squares. In: 19th International Conference on Pattern Recognition, December 2008, pp. 1-4 (2008)

9. Chandra, S., Svalbe, I., Guédon, J.P.: An exact, non-iterative Mojette inversion technique utilising ghosts. In: Coeurjolly, D., Sivignon, I., Tougne, L., Dupont, F. (eds.) DGCI 2008. LNCS, vol. 4992, pp. 401-412. Springer, Heidelberg (2008)

10. Servières, M., Normand, N., Guédon, J.P., Bizais, Y.: The Mojette transform: Discrete angles for tomography. In: Herman, G., Kuba, A. (eds.) Proceedings of the Workshop on Discrete Tomography and its Applications. Electronic Notes in Discrete Mathematics, vol. 20, pp. 587-606 (2005)

11. Guédon, J., Normand, N., Kingston, A., Parrein, B., Serviéres, M., Evenou, P., Svalbe, I., Autrusseau, F., Hamon, T., Bizais, Y., Coeurjolly, D., Boulos, F., Grail, E.: The Mojette Transform: Theory and Applications. ISTE-Wiley (2009)

12. Chen, X., Lu, S., Yuan, X.: Midpoint line algorithm for high-speed high-accuracy rotation of images. In: IEEE Conf. on Systems, Man and Cybernetics, Beijing, China, pp. 2739-2744 (1996) 